\title{
OPEN Synthesis and molecular structure of perhalogenated rhenium-oxo corroles
}

\author{
Abraham B. Alemayehu ${ }^{1}$, Rune F. Einrem ${ }^{1}$, Laura J. McCormick-McPherson ${ }^{2}$, \\ Nicholas S. Settineri ${ }^{2,3}$ \& Abhik Ghosh ${ }^{1}$
}

As part of our efforts to develop rhenium-oxo corroles as photosensitizers for oxygen sensing and photodynamic therapy, we investigated the potential B-perhalogenation of five ReO meso-tris(paraX-phenyl)corroles, $\operatorname{Re}[T p X P C](O)\left(X=\mathrm{CF}_{3}, \mathrm{H}, \mathrm{F}, \mathrm{CH}_{3}\right.$, and $\left.\mathrm{OCH}_{3}\right)$, with elemental chlorine and bromine. With $\mathrm{Cl}_{2}$, $b$-octachlorinated products $\mathrm{Re}\left[\mathrm{Cl}_{8} \mathrm{~T} p X P C\right](\mathrm{O})$ were rapidly obtained for $\mathrm{X}=\mathrm{CF}_{3}, \mathrm{H}$, and $\mathrm{CH}_{3}$, but $\mathrm{X}=\mathrm{OCH}_{3}$ resulted in overchlorination on the meso-aryl groups. Full b-octabromination proved slower relative to $\mathrm{Cu}$ and Ir corroles, but the desired $\operatorname{Re}\left[\mathrm{Br}_{8} \mathrm{~T} p \mathrm{XPC}\right](\mathrm{O})$ products were finally obtained for $\mathrm{X}=\mathrm{H}$ and $\mathrm{F}$ after a week at room temperature. For $\mathrm{X}=\mathrm{CH}_{3}$ and $\mathrm{OCH}_{3}$, these conditions led to undecabrominated products $\operatorname{Re}\left[\mathrm{Br}_{11} T p X P C\right](O)$. Compared to the $B$-unsubstituted starting materials, the $B$-octahalogenated products were found to exhibit sharp ${ }^{1} \mathrm{H}$ NMR signals at room temperature, indicating that the aryl groups are locked in place by the $B$-halogens, and substantially redshifted Soret and $Q$ bands. Single-crystal $X$-ray structures of $\operatorname{Re}\left[\mathrm{Cl}_{8} T p C F_{3} P C\right](O), \operatorname{Re}\left[\mathrm{Cl}_{8} T p \mathrm{CH}_{3} \mathrm{PC}\right](\mathrm{O})$, and $\operatorname{Re}\left[\mathrm{Br}_{8} \mathrm{~T} p \mathrm{FPC}\right](\mathrm{O})$ revealed mild saddling for one $\mathrm{Cl}_{8}$ structure and the $\mathrm{Br}_{8}$ structure. These structural variations, however, appear too insignificant to explain the slowness of the $b$-octabromination protocols, which seems best attributed to the deactivating influence of the high-valent Re center.

The remarkable $\beta$-octachlorination and $\beta$-octabromination of metallotetraarylporphyrins was first reported by the Traylor, Dolphin and their research groups in the $1980 \mathrm{~s}^{1,2}$. During the $1990 \mathrm{~s}$, iron and manganese complexes of $\beta$-octahalogenoporphyrins were intensively investigated as rugged, synthetic models of cytochrome $\mathrm{P} 450^{3-6}$. $\beta$-Octahalogeno-meso-tetraarylporphyrin derivatives also provided textbook examples of saddling, a nonplanar distortion in which the pyrrole rings are alternately tilted up and down relative to the mean porphyrin plane ${ }^{7-11}$. The compounds became the subject of a battery of spectroscopic, electrochemical, and structural studies, which yielded a rich body of insights on substituent effects in porphyrin derivatives ${ }^{10,12-15}$.

With the advent of simple, one-pot syntheses ${ }^{16-19}$ of corroles $^{20,21}, \beta$-octabromination was also found to work for certain corrole derivatives ${ }^{22,23}$. In our laboratory, we prepared some of the first $\beta$-octabromo-mesotriarylcorroles, initially the copper complexes ${ }^{24,25}$ and subsequently also the free bases ${ }^{26-28}$. Remarkably, a number of crystal structures of $\beta$-octabrominated metallocorroles revealed planar corrole rings, underscoring the rigidity of the corrole ring system relative to porphyrins ${ }^{23,29-31}$. Today we know that saddling in corroles is largely limited to copper ${ }^{32-37}$ corroles (and in part to silver ${ }^{38}$ corroles but not gold ${ }^{39-42}$ corroles), where it is thought to be a manifestation of ligand noninnocence, i.e., a distortion mode that facilitates $\mathrm{Cu}^{\mathrm{II}}\left(\mathrm{d}_{\mathrm{x} 2-\mathrm{y} 2}\right)$-corrole ${ }^{-2-}$ antiferromagnetic coupling ${ }^{43-46}$.

The present study is part of our ongoing efforts to functionalize and derivatize $5 \mathrm{~d}$ metallocorroles ${ }^{38-42,47-54}$. These complexes provide unusual examples of a large transition metal ion coordinated to a sterically constrained macrocyclic ligand. Despite the steric mismatch inherent in their structures, a good fraction of these complexes exhibit remarkable thermal, chemical, and photochemical stability. Many also exhibit near-IR phosphorescence and also efficiently sensitize singlet oxygen formation, which has led to applications in oxygen sensing, photodynamic therapy, and dye-sensitized solar cells ${ }^{55-63}$. The chemical reactivity of these complexes, by and large, remains poorly explored, with only a handful of reports on the subject. $\beta$-Octachlorination has been reported for an OsN corrole ${ }^{64}$, while gold corroles have been polyiodinated, with 4-5 iodines attached to the $\beta$-positions ${ }^{65,66}$. A couple of examples of metal-centered reactivity have also been documented; thus, $\mathrm{MoO}^{67}$ and $\mathrm{ReO}^{50}$ corroles have been transformed to the $\mathrm{MX}_{2}(\mathrm{X}=\mathrm{Cl}, \mathrm{Ph})$ derivatives, so-called Viking helmet corroles ${ }^{68}$, while OsN corroles

${ }^{1}$ Department of Chemistry, UiT - The Arctic University of Norway, 9037 Tromsø, Norway. ${ }^{2}$ Advanced Light Source, Lawrence Berkeley National Laboratory, Berkeley, CA 94720-8229, USA. ${ }^{3}$ Department of Chemistry, University of California, Berkeley, Berkeley, CA 94720, USA. ${ }^{\varpi}$ email: abhik.ghosh@uit.no 


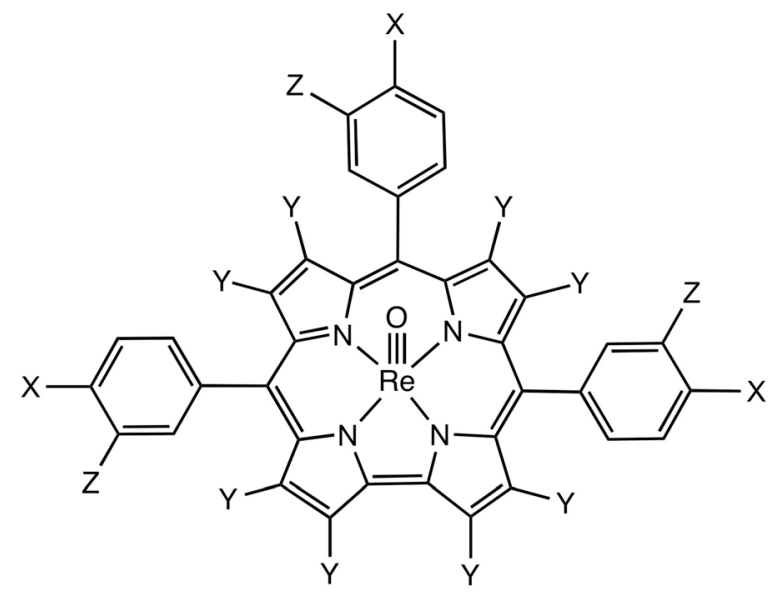

\begin{tabular}{|lccc|}
\hline Compound & $\mathrm{X}$ & $\mathrm{Y}$ & $\mathrm{Z}$ \\
\hline $\operatorname{Re}[\mathrm{T} p X \mathrm{XPC}](\mathrm{O})$ & $\mathrm{CF}_{3}, \mathrm{H}, \mathrm{F}, \mathrm{CH}_{3}, \mathrm{OCH}_{3}$ & $\mathrm{H}$ & $\mathrm{H}$ \\
$\operatorname{Re}\left[\mathrm{Cl}_{8} \mathrm{~T} p X \mathrm{PC}\right](\mathrm{O})$ & $\mathrm{CF}_{3}, \mathrm{H}, \mathrm{CH}_{3}$ & $\mathrm{Cl}$ & $\mathrm{H}$ \\
$\operatorname{Re}\left[\mathrm{Br}_{8} \mathrm{~T} p X P C\right](\mathrm{O})$ & $\mathrm{H}, \mathrm{F}$ & $\mathrm{Br}$ & $\mathrm{H}$ \\
$\operatorname{Re}\left[\mathrm{Br}_{11} \mathrm{~T} p X P C\right](\mathrm{O})$ & $\mathrm{CH}_{3}, \mathrm{OCH}_{3}$ & $\mathrm{Br}$ & $\mathrm{Br}$ \\
\hline
\end{tabular}

Figure 1. ReO corroles synthesized in this work.

have been found to act as unusual $\pi$-acceptor metallaligands toward $\mathrm{Pt}(\mathrm{II})^{64}$. Herein we document our efforts to halogenate rhenium(V)-oxo triarylcorroles with elemental chlorine and bromine (Fig. 1).

The main contributions of this work may be described as threefold. First and foremost are the products themselves, which should serve as starting materials in a variety of cross-coupling reactions, affording, for example, $\mathrm{ReO}$ undecaarylcorroles via the Suzuki-Miyaura reaction. The products thus obtained are likely to further extend the growing range of applications of $\mathrm{ReO}$ corroles $^{60,61}$. Second, three of the $\beta$-octahalogenated products have yielded single-crystal X-ray structures, shedding light on potential distortion modes available to these sterically congested species. Third, although the "messy" reaction conditions did not allow us to devise kinetic studies, qualitative observations indicate major differences in the times required for $\beta$-octabromination as a function of the coordinated metal, which appear to be ascribable to the electronic effects of the coordinated metal, as described below.

\section{Results and discussion}

Synthetic method development. Optimizing the conditions for $\beta$-octachlorination proved relatively straightforward ${ }^{69}$. In the final, optimized protocol, a saturated, greenish-yellow solution of chlorine $\left(\mathrm{Cl}_{2}\right)$ in chloroform was added dropwise, over a period of $20 \mathrm{~min}$, to a benzene solution of $\beta$-unsubstituted ReO corroles, $\operatorname{Re}[\mathrm{T} p \mathrm{XPC}](\mathrm{O})$, maintained at $0{ }^{\circ} \mathrm{C}$ in an ice bath. The ice bath was removed after $1 \mathrm{~h}$ and the reaction was allowed to continue at room temperature for $24 \mathrm{~h}$. After work-up, HR-ESI mass spectrometry and ${ }^{1} \mathrm{H}$ NMR spectroscopy showed that all eight $\beta$-hydrogens had been fully replaced by chlorine atoms for $\mathrm{X}=\mathrm{CF}_{3}, \mathrm{H}$ and $\mathrm{CH}_{3}$. For $\mathrm{X}=\mathrm{OCH}_{3}$, the most electron-donating substituent, however, overchlorination was observed, with $\mathrm{Cl}_{8}$, $\mathrm{Cl}_{9}$ and $\mathrm{Cl}_{10}$ products appearing in a ratio of approximately 15:100:80 (see Figure S11 in the Supplementary Material).

Finding the optimum conditions for $\beta$-octabromination, in contrast, involved a fair amount of trial and error. Initial experiments with up to 100 equiv liquid bromine $\left(\mathrm{Br}_{2}\right)$ in chloroform led after $4 \mathrm{~h}$ to a mixture of $\mathrm{Br}_{4}, \mathrm{Br}_{5}$, $\mathrm{Br}_{6}$, and $\mathrm{Br}_{7}$ products, with only traces of $\mathrm{Br}_{8}$. Increasing the reaction time to $16 \mathrm{~h}$ also led to the same complex mixtures. Increasing the reaction time to $48 \mathrm{~h}$, however, led to the selective formation of $\mathrm{Br}_{6}$ and $\mathrm{Br}_{7}$ derivatives as the major products, with the $\mathrm{Br}_{8}$ appearing as a minor product. We surmised that increasing the concentration of elemental bromine and lengthening the reaction time even further might result in full $\beta$-octabromination. Accordingly, we increased the amount of elemental bromine threefold and extended the reaction time to 7 days. As before, we began with 100 equiv of $\mathrm{Br}_{2}$ added dropwise over a 20 -min period. On the 2 nd day, we added another 100 equiv of $\mathrm{Br}_{2}$ dropwise over $20 \mathrm{~min}$. We did the same on the $3 \mathrm{rd}$ day and let the reaction run for an additional 4 days, i.e., a total of 7 days. After work up, HR-MS and ${ }^{1} \mathrm{H}$ NMR showed that octabrominated complexes $\operatorname{Re}\left[\mathrm{Br}_{8} \mathrm{~T} p \mathrm{XPC}\right](\mathrm{O})$ had cleanly formed for $\mathrm{X}=\mathrm{H}$ and $\mathrm{F}$. For $\mathrm{X}=\mathrm{CH}_{3}$ and $\mathrm{OCH}_{3}$, on the other hand, over-bromination had occurred, resulting in the undecabrominated complexes $\operatorname{Re}\left[\mathrm{Br}_{11} \mathrm{~T} p \mathrm{XPC}\right](\mathrm{O})$.

The long times needed for $\beta$-octabromination of $\mathrm{ReO}$ corroles, and presumably also for OsN corroles, may be contrasted with the rapid reactions observed for $\mathrm{Cu}^{24}$ and $\mathrm{Ir}^{23}$ corroles. The difference is most simply ascribed to the higher oxidation state of the central metal in the case of the $\mathrm{ReO}$ and $\mathrm{OsN}$ complexes, which presumably 


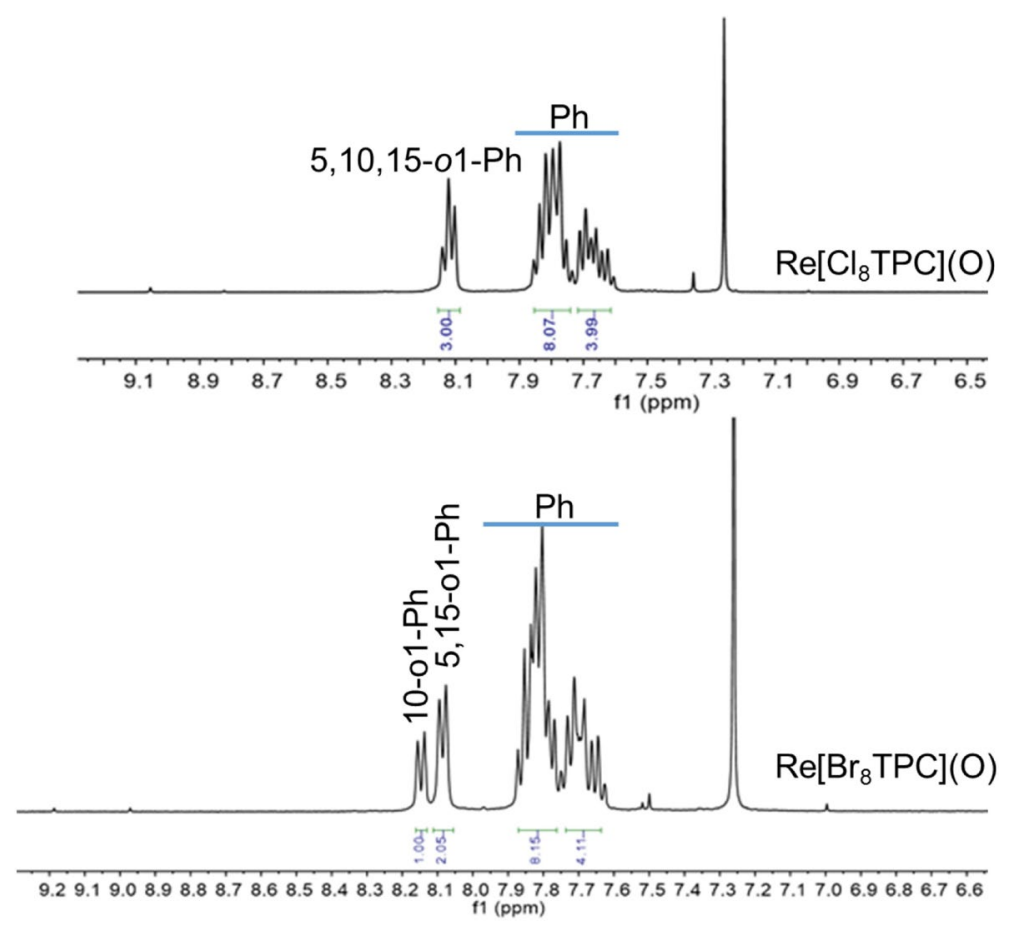

Figure 2. ${ }^{1} \mathrm{H}$ NMR spectra of $\operatorname{Re}\left[\mathrm{Cl}_{8} \mathrm{TPC}\right](\mathrm{O})$ (top) and $\mathrm{Re}\left[\mathrm{Br}_{8} \mathrm{TPC}\right](\mathrm{O})$ (bottom) in $\mathrm{CDCl}_{3}$ at $298 \mathrm{~K}$.

deactivates the corrole toward electrophilic substitution. Such a rationale is in line with the redox potentials of the metallocorroles; the oxidation potentials of $\mathrm{ReO}^{50}$ and $\mathrm{OsN}^{52}$ corroles are substantially higher than those of analogous $\mathrm{Cu}^{24,38}$ and $\operatorname{Ir}^{23}$ corroles.

${ }^{1}$ H NMR and UV-Vis spectroscopy. Both types of spectra clearly reflect the effect of $\beta$-octahalogenation. The most obvious change in the ${ }^{1} \mathrm{H}$ NMR spectra is associated with the disappearance of the $\beta$-proton signals between 8.5 and $10 \mathrm{ppm}$ (Fig. 2). Another highly characteristic change is that unlike the room-temperature ${ }^{1} \mathrm{H}$ NMR spectra of starting complexes ${ }^{50}$, the spectra of the $\beta$-perhalogenated products are already sharp at room temperature. The broad ${ }^{1} \mathrm{H}$ NMR spectra of $\operatorname{Re}[\mathrm{T} p \mathrm{XPC}](\mathrm{O})$ at room temperature reflect partially restricted rotation of the meso-aryl groups and only around $-20{ }^{\circ} \mathrm{C}$ or so do the aryl ortho and meta signals split into distinguishable $o, o^{\prime}$ and $m, m^{\prime}$ signals. In $\beta$-perhalogenated products, the aryls are effectively locked in place even at room temperature.

Like a number of other classes of $5 \mathrm{~d}$ metallocorroles ${ }^{40,51,52,54}$, ReO triarylcorroles exhibit sharp, intense Soret bands and characteristic, double-humped Q bands ${ }^{50}$. The qualitative shapes of these features persist relatively unaltered upon $\beta$-octahalogenation. $\beta$-Octahalogenation does, however, engender significant redshifts for each of these features. Thus, for $\beta$-octachlorination, the Soret and Q bands redshift by around 9 and $13-16 \mathrm{~nm}$, respectively, while for $\beta$-octabomination, the corresponding shifts are 17-19 and 21-22 nm, respectively (Table 1 and Fig. 3).

X-ray crystallography and molecular structure. The molecular structures of $\beta$-perhalogenated ReO corroles were expected to be of unusual interest as a window into potential deformation pathways of the corrole macrocycle in response to extreme peripheral crowding ${ }^{70}$. In general, $\beta$-octahalogenation does not result in significant nonplanar deformations for metallocorroles, reflecting the rigidity of the direct pyrrole-pyrrole linkage ${ }^{23,28-31}$. Coinage metal corroles, especially $\mathrm{Cu}^{32-37}$ corroles but also $\mathrm{Ag}^{38}$ corroles, constitute the major exceptions to this generalization. These metallocorroles are intrinsically saddled, as a result of a specific metal(d)$\operatorname{corrole}(\pi)$ orbital interaction, which results in a noninnocent, partial $\mathrm{M}^{\mathrm{II}}$-corrole ${ }^{-2-}$ character of the complexes. Importantly, the degree of saddling in Cu corroles, while substantial even in $\beta$-unsubstituted triarylcorrole derivatives, can be greatly enhanced by $\beta$-octasubstitution. The same orbital interaction, however, is energetically unfavorable for Au corroles ${ }^{40}$. Accordingly, even undecasubstituted Au corroles are fairly rigorously planar ${ }^{29,30,38,40-42}$. So, as a matter of fact, are six-coordinate Ir corroles, including $\operatorname{Ir} \beta$-octabrominated derivatives ${ }^{23,56,62}$. Diboron corroles provide another example of a dramatic structural influence of $\beta$-octasubstitution. Thus, while simple corroles yield strongly domed complexes with cisoid $\mathrm{FBOBF}$ groups ${ }^{71}, \beta$-octabromo-meso-triarylcorroles yield unbridged bis- $\mathrm{BF}_{2}$ complexes, with the $\mathrm{BF}_{2}$ groups on opposite sides of corrole macrocycle $\mathrm{e}^{72}$.

Three of the products obtained here, including one octabrominated and two octachlorinated products, yielded single-crystal X-ray structures (Table 2). The $\mathrm{Re}-\mathrm{O}$ and $\mathrm{Re}-\mathrm{N}$ bond distances, as well as the displacement of the Re atom above the mean $\mathrm{N}_{4}$ plane, all turned out to be essentially identical to those observed for $\beta$-unsubstituted

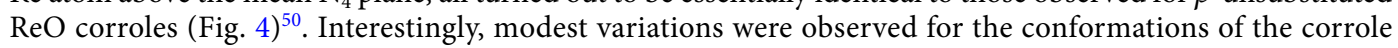




\begin{tabular}{|l|l|l|l|l|l|}
\hline Compound & $\mathbf{N}$ & Soret & $\mathbf{Q}_{1}$ & $\mathbf{Q}_{2}$ & Ref \\
\hline $\operatorname{Re}\left[\mathrm{T} p \mathrm{CF}{ }_{3} \mathrm{PC}\right](\mathrm{O})$ & $320(1.77)$ & $438(10.74)$ & $552(1.63)$ & $585(1.99)$ & 50 \\
\hline $\operatorname{Re}[\mathrm{TPC}](\mathrm{O})$ & $320(1.64)$ & $439(10.09)$ & $552(1.99)$ & $585(2.34)$ & 50 \\
\hline $\operatorname{Re}[\mathrm{T} p \mathrm{FPC}](\mathrm{O})$ & $319(1.57)$ & $438(10.16)$ & $553(1.53)$ & $585(1.93)$ & 50 \\
\hline $\operatorname{Re}\left[\mathrm{T} p \mathrm{CH}_{3} \mathrm{PC}\right](\mathrm{O})$ & $318(2.92)$ & $440(11.18)$ & $555(1.86)$ & $587(2.37)$ & 50 \\
\hline $\operatorname{Re}\left[\mathrm{Cl}_{8} \mathrm{~T} \mathrm{CF}_{3} \mathrm{PC}\right](\mathrm{O})$ & $349(2.17)$ & $447(11.04)$ & $565(1.58)$ & $599(2.24)$ & This work \\
\hline $\operatorname{Re}\left[\mathrm{Cl}_{8} \mathrm{TPC}\right](\mathrm{O})$ & $349(2.04)$ & $448(9.93)$ & $567(1.41)$ & $599(2.11)$ & This work \\
\hline $\operatorname{Re}\left[\mathrm{Cl}_{8} \mathrm{~T} p \mathrm{CH}_{3} \mathrm{PC}\right](\mathrm{O})$ & $350(2.54)$ & $449(11.00)$ & $568(1.70)$ & $601(2.47)$ & This work \\
\hline $\operatorname{Re}\left[\mathrm{Br}_{8} \mathrm{~T} p \mathrm{FPC}\right](\mathrm{O})$ & $357(2.08)$ & $456(9.77)$ & $574(1.43)$ & $607(2.12)$ & This work \\
\hline $\operatorname{Re}\left[\mathrm{Br}_{8} \mathrm{TPC}\right](\mathrm{O})$ & $357(2.28)$ & $456(10.45)$ & $575(1.59)$ & $607(2.40)$ & This work \\
\hline $\operatorname{Re}\left[\mathrm{Br}_{11} \mathrm{~T} p \mathrm{CH} H_{3} \mathrm{PC}\right](\mathrm{O})$ & $359(2.07)$ & $458(9.70)$ & $574(1.44)$ & $608(2.10)$ & This work \\
\hline $\operatorname{Re}\left[\mathrm{Br}_{11} \mathrm{~T} p \mathrm{OCH}{ }_{3} \mathrm{PC}\right](\mathrm{O})$ & $356(1.15)$ & $459(8.59)$ & $574(1.15)$ & $608(1.72)$ & This work \\
\hline
\end{tabular}

Table 1. UV-Vis spectral data in dichloromethane, $\lambda_{\max }$ and $\varepsilon \times 10^{-4}\left(\mathrm{M}^{-1} \mathrm{~cm}^{-1}\right)$, for the compounds studied.

(a)

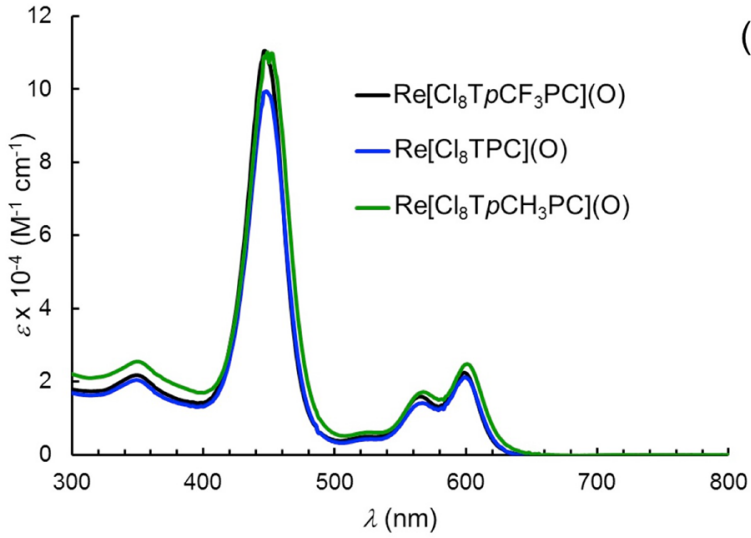

(c)

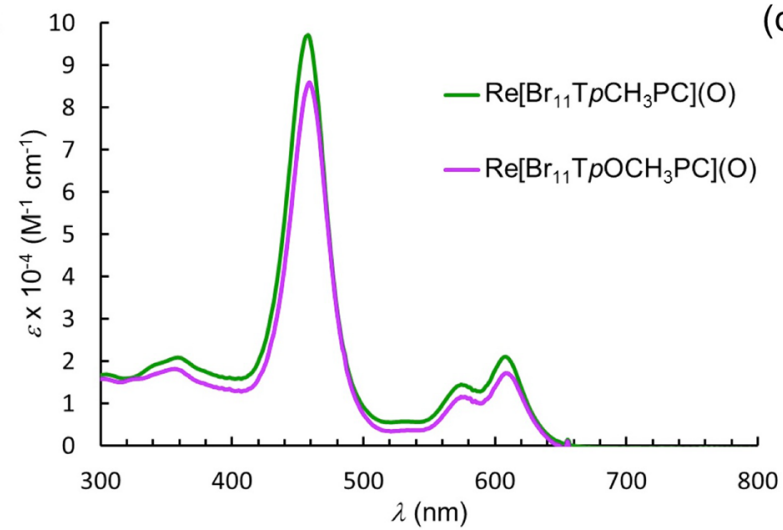

(b)

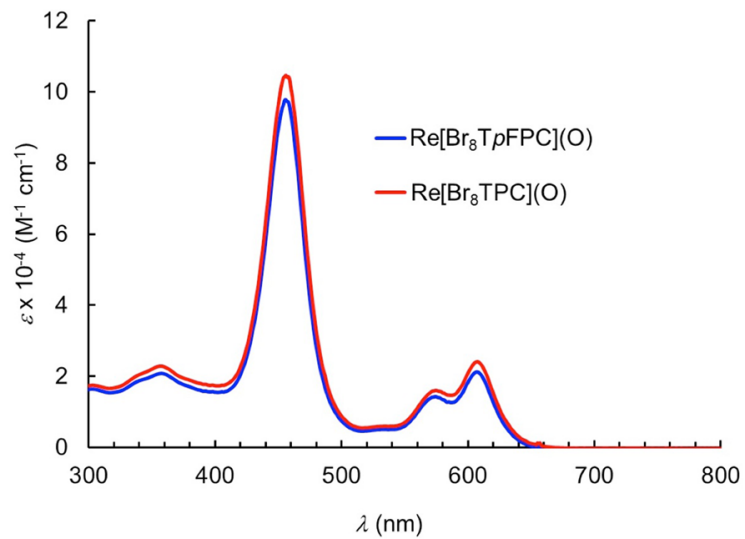

(d)

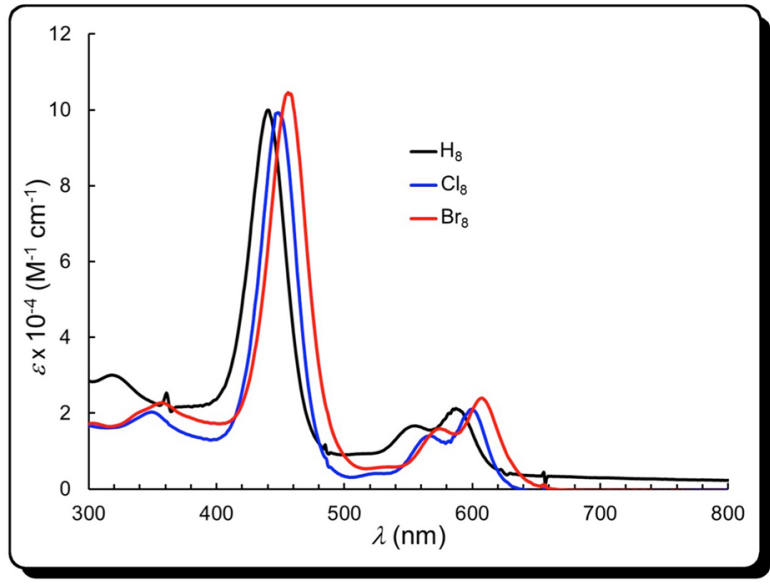

Figure 3. UV-Vis spectra in dichloromethane for (a) $\mathrm{Re}\left[\mathrm{Cl}_{8} \mathrm{~T} p \mathrm{XPC}\right](\mathrm{O})\left(\mathrm{X}=\mathrm{CF}_{3}, \mathrm{H}\right.$ and $\left.\mathrm{CH}_{3}\right),(\mathbf{b})$ $\operatorname{Re}\left[\mathrm{Br}_{8} \mathrm{~T} p \mathrm{XPC}\right](\mathrm{O})(\mathrm{X}=\mathrm{F}$ and $\mathrm{H})$ and $(\mathbf{c}) \operatorname{Re}\left[\mathrm{Br}_{11} \mathrm{~T} p \mathrm{XPC}\right](\mathrm{O})\left(\mathrm{X}=\mathrm{CH}_{3}\right.$ and $\left.\mathrm{OCH}_{3}\right)$. (d) Comparative UV-Vis spectra for $\operatorname{Re}[\mathrm{TPC}](\mathrm{O})$ (black), $\operatorname{Re}\left[\mathrm{Cl}_{8} \mathrm{TPC}\right](\mathrm{O})$ (blue), and $\operatorname{Re}\left[\mathrm{Br}_{8} \mathrm{TPC}\right](\mathrm{O})$ (red).

macrocycles. Aligning the mean $\mathrm{N}_{4}$ planes of $\beta-\mathrm{H}_{8}, \beta-\mathrm{Cl}_{8}$, and $\beta-\mathrm{Br}_{8}$ structures showed that the corrole macrocycles in these systems might be described as slightly domed, planar, and slightly, if somewhat irregularly, saddled, respectively (Fig. 5 and Table 3 ). The $\beta$ - $\mathrm{Br}_{8}$ crystal structure reported here thus represents a rare example of a saddled corrole, aside from the coinage metal corroles.

Might the above structural differences play a role in explaining the slow rates of $\beta$-octabromination of $\mathrm{ReO}$ triarylcorroles relative to $\mathrm{Cu}$ and Ir triarylcorroles? Given that the above differences are rather minor (Table 3), we believe that the answer is essentially 'no'; as stated above, the high oxidation state of the Re center provides the most plausible explanation for the slowness of the octabromination. 


\begin{tabular}{|c|c|c|c|}
\hline Sample & $\operatorname{Re}\left[\mathrm{Cl}_{8} \mathrm{~T} p \mathrm{CF}_{3} \mathrm{PC}\right](\mathrm{O})$ & $\operatorname{Re}\left[\mathrm{Cl}_{8} \mathrm{~T} p \mathrm{CH}_{3} \mathrm{PC}\right](\mathrm{O})$ & $\operatorname{Re}\left[\mathrm{Br}_{8} \mathrm{~T} p \mathrm{FPC}\right](\mathrm{O})$ \\
\hline Chemical formula & $\mathrm{C}_{40} \mathrm{H}_{12} \mathrm{Cl}_{8} \mathrm{~F}_{9} \mathrm{~N}_{4} \mathrm{O} \mathrm{Re}$ & $\mathrm{C}_{40} \mathrm{H}_{21} \mathrm{Cl}_{8} \mathrm{~N}_{4} \mathrm{O} \mathrm{Re}$ & $\mathrm{C}_{37} \mathrm{H}_{12} \mathrm{Br}_{8} \mathrm{~F}_{3} \mathrm{~N}_{4} \mathrm{O} \mathrm{Re}$ \\
\hline Formula mass & 1203.78 & 1041.87 & 1411.38 \\
\hline Crystal system & Triclinic & Triclinic & Triclinic \\
\hline Crystal size $\left(\mathrm{mm}^{3}\right)$ & $0.200 \times 0.170 \times 0.150$ & $0.050 \times 0.030 \times 0.010$ & $0.080 \times 0.080 \times 0.030$ \\
\hline Space group & $P-1$ & $P-1$ & $P-1$ \\
\hline$\lambda(\AA)$ & 0.7288 & 0.7288 & 0.7288 \\
\hline$a(\AA)$ & $16.0327(12)$ & $12.5075(5)$ & $11.7273(4)$ \\
\hline$b(\AA)$ & 17.3203(13) & $12.9074(6)$ & $14.1820(5)$ \\
\hline$c(\AA)$ & $17.8739(13)$ & $13.7640(5)$ & $14.6880(5)$ \\
\hline$\alpha(\operatorname{deg})$ & $116.0080(10)$ & $74.8780(10)$ & $100.8390(10)$ \\
\hline$\beta(\operatorname{deg})$ & $90.679(2)$ & $85.9610(10)$ & $106.6030(10)$ \\
\hline$\gamma(\operatorname{deg})$ & $102.117(2)$ & $82.5010(10)$ & $90.7700(10)$ \\
\hline$Z$ & 2 & 2 & 2 \\
\hline$V\left(\AA^{3}\right)$ & $4330.8(6)$ & 2125.22(15) & 2293.38(14) \\
\hline Temperature (K) & $100(2)$ & $100(2)$ & $100(2)$ \\
\hline Density $\left(\mathrm{g} / \mathrm{cm}^{3}\right)$ & 1.998 & 1.765 & 2.269 \\
\hline Measured reflections & 108,021 & 59,434 & 151,248 \\
\hline Unique reflections & 25,785 & 8713 & 11,449 \\
\hline Parameters & 1231 & 567 & 602 \\
\hline Restraints & 59 & 9 & 6 \\
\hline$R_{\text {int }}$ & 0.0685 & 0.0511 & 0.0405 \\
\hline$\theta$ range (deg.) & $1.341-31.393$ & $1.573-27.142$ & $2.027-29.131$ \\
\hline$R_{1}, w R_{2}$ all data & $0.0780,0.1432$ & $0.0332,0.0794$ & $0.0259,0.0526$ \\
\hline$S($ GooF) all data & 1.112 & 1.075 & 1.039 \\
\hline Max/min res. dens. $\left(e / \AA^{3}\right)$ & $2.370 /-2.057$ & $2.163 /-1.534$ & $1.714 /-1.377$ \\
\hline
\end{tabular}

Table 2. Crystallographic data for the complexes analyzed.

(a)

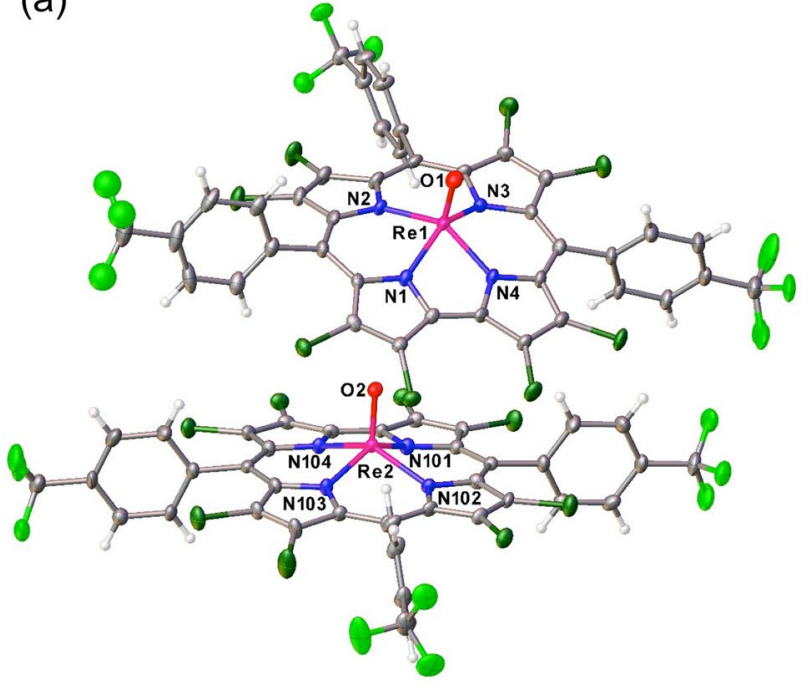

(b)

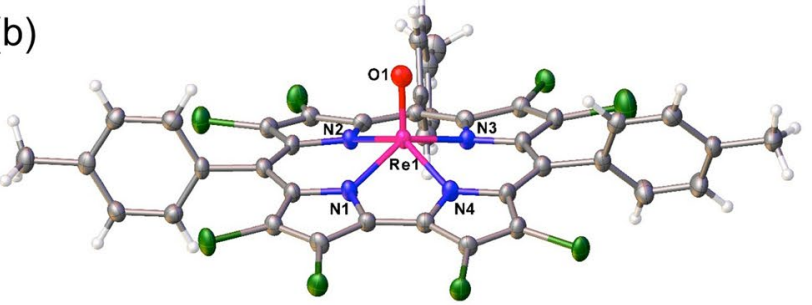

(c)

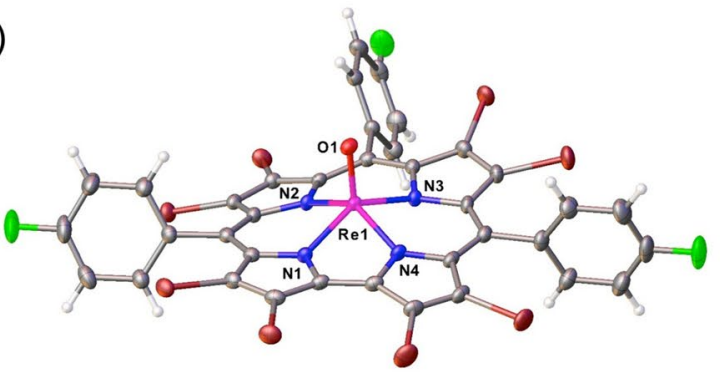

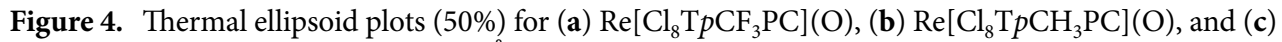
$\operatorname{Re}\left[\mathrm{Br}_{8} \mathrm{~T} p \mathrm{FPC}\right](\mathrm{O})$. Selected distances $(\AA ̊)$ for $(\mathbf{a}) \operatorname{Re}\left[\mathrm{Cl}_{8} \mathrm{~T}_{p} \mathrm{CF}_{3} \mathrm{PC}\right](\mathrm{O})$ : Re1-N1 1.997(5), Re1-N2 2.015(4), Re1-N3 2.025(4), Re1-N4 1.995(4), and Re1-O1 1.670(4); Re2-N101 1.989(4), Re2-N102 2.014(4), Re2N103 2.019(4), Re2-N104 1.980(4), Re2-O2 1.672. Selected distances ( $\AA$ ) for Re[Cl $\left.\mathrm{Cl}_{8} \mathrm{TCH}_{3} \mathrm{PC}\right](\mathrm{O})$ : Re1-N1 1.995(3), Re1-N2 2.019(3), Re1-N3 2.017(3), Re1-N4 1.992(3), and Re1-O1 1.677(3). Selected distances ( $\AA$ ) for $\mathrm{Re}\left[\mathrm{Br}_{8} \mathrm{~T} p \mathrm{FPC}\right](\mathrm{O})$ : Re1-N1 1.996(2), Re1-N2 2.019(2), Re1-N3 2.011(2), Re1-N4 1.997(2), and Re1-O1 $1.673(2)$. 


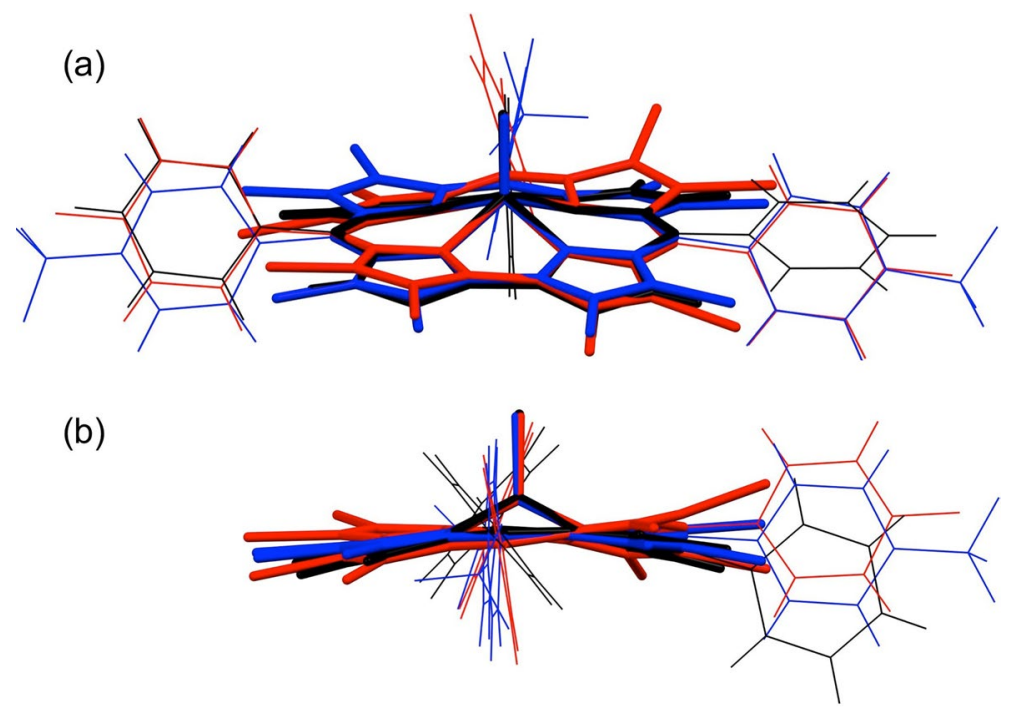

Figure 5. Mercury overlay of the nitrogen atoms of $\operatorname{Re}[\mathrm{TPC}](\mathrm{O})$ (black), molecule 1 of $\operatorname{Re}\left[\mathrm{Cl}_{8} \mathrm{~T}_{p} \mathrm{CF}_{3} \mathrm{PC}\right](\mathrm{O})$ (blue) and $\operatorname{Re}\left[\mathrm{Br}_{8} \mathrm{~T} p \mathrm{FPC}\right](\mathrm{O})$. (a) View from above C1-C19 toward C10. (b) View along C5-C15.

\begin{tabular}{|l|l|l|l|}
\hline Molecule & $\operatorname{Re}-\mathbf{N}_{4}(\AA)$ & $\chi_{\text {C8-C9-C11-C12 }}\left({ }^{\circ}\right)$ & $\phi_{\text {C5-Re-C15 }}\left({ }^{\circ}\right)$ \\
\hline $\operatorname{Re}[\mathrm{TPC}](\mathrm{O})$ & 0.704 & 7.2 & 155.1 \\
\hline $\operatorname{Re}\left[\mathrm{Cl}_{8} \mathrm{~T} p \mathrm{CF}{ }_{3} \mathrm{PC}\right](\mathrm{O}) ;$ molecule 1 & 0.671 & 15.4 & 155.1 \\
\hline $\operatorname{Re}\left[\mathrm{Cl}_{8} \mathrm{~T}_{p} \mathrm{CF}_{3} \mathrm{PC}\right](\mathrm{O}) ;$ molecule 2 & 0.659 & 6.7 & 154.0 \\
\hline $\operatorname{Re}\left[\mathrm{Cl}_{8} \mathrm{~T} p \mathrm{CH}_{3} \mathrm{PC}\right](\mathrm{O})$ & 0.671 & 8.5 & 157.4 \\
\hline $\operatorname{Re}\left[\mathrm{Br}_{8} \mathrm{~T} p \mathrm{FPC}\right](\mathrm{O})$ & 0.668 & 11.9 & 152.5 \\
\hline
\end{tabular}

Table 3. Measures of nonplanarity for Re corroles: the Re-N4 displacement ( $\AA$ ), the C8-C9-C11-C12 saddling dihedral $\left({ }^{\circ}\right)$, and the C5-Re-C15 angle $\left(^{\circ}\right)$.

\section{Conclusion}

In summary, we have optimized reaction conditions leading to $\beta$-perhalogenation of ReO triarylcorroles with elemental chlorine and bromine. $\beta$-Perhalogenation is accompanied by highly characteristic changes in the ${ }^{1} \mathrm{H}$ NMR and UV-Vis spectra of the compounds. Three of the $\beta$-octahalogenated products, including two of the octachlorinated complexes and one octabrominated complex, yielded single-crystal X-ray structures. On the whole, the structures were remarkably similar to those of $\beta$-unsubstituted $\mathrm{ReO}$ corroles. Minor variations were observed in regard to macrocycle conformation. Thus, whereas $\beta$-unsubstituted ReO corroles are generally slightly domed, one octachlorinated complex and the octabrominated complex were found to exhibit slightly saddled macrocycles. These structural differences, however, appear to be too minor to explain the comparative slowness of $\beta$-octabromination of $\mathrm{ReO}$ triarylcorroles, relative to their $\mathrm{Cu}$ and $\mathrm{Ir}$ counterparts. The slowness is more plausibly attributed to the high oxidation state of the Re center, which leads to a higher oxidation potential for the corrole macrocycle and, in turn, a lower susceptibility to electrophilic attack. It is hoped that the $\beta$-perhalogenated complexes reported herein will act as substrates in Suzuki-Miyaura and other palladiumcatalyzed transformations, thereby affording additional avenues for the elaboration of $\mathrm{ReO}$ corroles.

\section{Experimental section}

Materials. Rhenium-oxo meso-triarylcorroles, $\operatorname{Re}[\mathrm{T} p \mathrm{XPC}](\mathrm{O})$, were synthesized as previously reported ${ }^{50}$. Chlorine gas $\left(\mathrm{Cl}_{2}\right)$, liquid bromine $\left(\mathrm{Br}_{2}\right)$, benzene, and chloroform were purchased from Sigma-Aldrich. Silica gel 60 (0.04-0.063 mm particle size, 230-400 mesh, Merck) was used for flash chromatography and silica gel 60 preparative thin-layer chromatography (PTLC) plates $(20 \mathrm{~cm} \times 20 \mathrm{~cm}, 0.5 \mathrm{~mm}$ thick; Merck) were used for final purification of all complexes.

Instrumental methods. UV-visible spectra were recorded on an HP 8453 spectrophotometer. ${ }^{1} \mathrm{H}$ NMR spectra $\left(298 \mathrm{~K}, \mathrm{CDCl}_{3}\right)$ were recorded on a $400 \mathrm{MHz}$ Bruker Avance III HD spectrometer equipped with a $5-\mathrm{mm}$ $\mathrm{BB} / 1 \mathrm{H}$ SmartProbe and referenced to residual $\mathrm{CHCl}_{3}$ at $7.26 \mathrm{ppm}$. High-resolution electrospray-ionization (HRESI) mass spectra were recorded from methanolic solution on an LTQ Orbitrap XL spectrometer. 
General procedure for the synthesis of $\operatorname{Re}\left[\mathrm{Cl}_{8} \mathrm{TpXPC}\right](\mathrm{O})$. To a $10-\mathrm{mL}$ benzene solution of $\operatorname{Re}[\mathrm{T} p \mathrm{XPC}](\mathrm{O})\left(\mathrm{X}=\mathrm{CF}_{3}: 15 \mathrm{mg}, 0.016 \mathrm{mmol} ; \mathrm{X}=\mathrm{H}: 25 \mathrm{mg}, 0.034 \mathrm{mmol} ; \mathrm{X}=\mathrm{CH}_{3}: 20 \mathrm{mg}, 0.026 \mathrm{mmol}\right)$ chilled to $0{ }^{\circ} \mathrm{C}$ in an ice-bath, was added a greenish-yellow, saturated solution of chlorine $\left(\mathrm{Cl}_{2}, 10 \mathrm{~mL}\right)$ in chloroform over a period of $20 \mathrm{~min}$. After an hour at $0{ }^{\circ} \mathrm{C}$, the ice-bath was removed and the reaction was allowed to continue under stirring at room temperature for a total of $24 \mathrm{~h}$. The reaction mixture was then quenched by washing twice with $20 \%$ aqueous sodium metabisulfite solution $(20 \mathrm{~mL} \times 2)$. The organic phase was thoroughly washed with distilled water, dried with sodium sulfate, and rotary-evaporated to dryness. The resulting crude product was dissolved in a minimum amount of dichloromethane and loaded onto a silica gel column prepared with 3:1 hexane/dichloromethane and eluted with the same solvent system. The resulting greenish-red product was collected, evaporated to dryness, and further purified via preparative thin-layer chromatography with the same solvent system. Yields and analytical details of new compounds are given below. X-ray-quality crystals of $\mathrm{Re}\left[\mathrm{Cl}_{8} \mathrm{~T} p \mathrm{CF}_{3} \mathrm{PC}\right](\mathrm{O})$ and $\mathrm{Re}\left[\mathrm{Cl}_{8} \mathrm{~T} p \mathrm{CH}_{3} \mathrm{PC}\right](\mathrm{O})$ were obtained by slow diffusion of methanol vapor into concentrated dichloromethane solutions of the complexes.

$\operatorname{Re}\left[\mathrm{Cl}_{8} \mathrm{TpCF} \mathrm{CF}_{3} \mathrm{PC}(\mathrm{O})\right.$. Yield $11.5 \mathrm{mg}$ (59.22\%). UV-Vis $\left(\mathrm{CH}_{2} \mathrm{Cl}_{2}\right) \lambda_{\max }\left[\mathrm{nm}, \varepsilon \times 10^{-4}\left(\mathrm{M}^{-1} \mathrm{~cm}^{-1}\right)\right]: 349$ (2.17), 447 (11.04), 565 (1.58), 599 (2.24). ${ }^{1} \mathrm{H} \mathrm{NMR}\left(400 \mathrm{MHz}, 25^{\circ} \mathrm{C}\right) \delta: 8.29$ (d, 3H, $\left.{ }^{3} \mathrm{~J}_{\mathrm{HH}}=7.92 \mathrm{~Hz}, 5,10,15-o 1-\mathrm{Ph}\right) ; 8.09$ $\left(\mathrm{d}, 2 \mathrm{H},{ }^{3} J_{\mathrm{HH}}=7.96 \mathrm{~Hz}, 5,15-o 2-\mathrm{Ph}\right) ; 8.05\left(\mathrm{~d}, 1 \mathrm{H},{ }^{3} \mathrm{~J}_{\mathrm{HH}}=7.60 \mathrm{~Hz}, 10-o 2-\mathrm{Ph}\right) ; 7.98\left(\mathrm{~d}, 4 \mathrm{H},{ }^{3} J_{\mathrm{HH}}=6.40 \mathrm{~Hz}, 5,15-m 1 \&\right.$ $m 2-\mathrm{Ph}) ; 7.92\left(\mathrm{~d}, 1 \mathrm{H},{ }^{3} J_{\mathrm{HH}}=9.80 \mathrm{~Hz}, 10-m 1-\mathrm{Ph}\right) ; 7.84\left(\mathrm{~d}, 1 \mathrm{H},{ }^{3} J_{\mathrm{HH}}=8.00 \mathrm{~Hz}, 10-m 2-\mathrm{Ph}\right)$. Elemental analysis calcd for $\mathrm{C}_{40} \mathrm{H}_{12} \mathrm{ON}_{4} \mathrm{~F}_{9} \mathrm{Cl}_{8} \mathrm{Re}$ : C 39.86, $\mathrm{H}$ 1.00, N 4.65; found: C 40.27, $\mathrm{H}$ 1.21. N 4.24. MS (ESI): $\mathrm{M}^{+}=1203.7915$ (expt), 1203.7880 (calcd for $\mathrm{C}_{40} \mathrm{H}_{12} \mathrm{ON}_{4} \mathrm{~F}_{9} \mathrm{Cl}_{8} \mathrm{Re}$ ).

$\operatorname{Re}\left[\mathrm{Cl}_{8} \mathrm{TPC}\right](\mathrm{O})$. Yield $23 \mathrm{mg}(66.71 \%)$. UV-Vis $\left(\mathrm{CH}_{2} \mathrm{Cl}_{2}\right) \lambda_{\max }\left[\mathrm{nm}, \varepsilon \times 10^{-4}\left(\mathrm{M}^{-1} \mathrm{~cm}^{-1}\right)\right]: 349$ (2.04), 448 (9.93), $567(1.41), 599(2.11) .{ }^{1} \mathrm{H}$ NMR $\left(400 \mathrm{MHz}, 25^{\circ} \mathrm{C}\right): \delta 8.12\left(\mathrm{~d}, 3 \mathrm{H},{ }^{3} J_{\mathrm{HH}}=7.48 \mathrm{~Hz}, 5,10,15-o 1-\mathrm{Ph}\right) ; 7.85-7.73$ $(\mathrm{m}, 8 \mathrm{H}, \mathrm{Ph}) ; 7.70-7.60(\mathrm{~m}, 4 \mathrm{H}, \mathrm{Ph})$. Elemental analysis calcd for $\mathrm{C}_{37} \mathrm{H}_{15} \mathrm{ON}_{4} \mathrm{Cl}_{8} \mathrm{Re}$ : C 44.38, H 1.51, N 5.60; found; $\mathrm{C} 44.07, \mathrm{H} 1.37, \mathrm{~N}$ 5.18. MS (ESI): $\mathrm{M}^{+}=999.8272$ (expt), 999.8258 (calcd for $\mathrm{C}_{37} \mathrm{H}_{15} \mathrm{ON}_{4} \mathrm{Cl}_{8} \mathrm{Re}$ ).

$\operatorname{Re}\left[\mathrm{Cl}_{8} \mathrm{~T} p \mathrm{CH}_{3} \mathrm{PC}\right](\mathrm{O})$. Yield $15.3 \mathrm{mg}(56.39 \%)$. UV-Vis $\left(\mathrm{CH}_{2} \mathrm{Cl}_{2}\right) \lambda_{\max }\left[\mathrm{nm}, \varepsilon \times 10^{-4}\left(\mathrm{M}^{-1} \mathrm{~cm}^{-1}\right)\right]: 350$ (2.54), 449 (11.00), 568 (1.70), 601 (2.47). ${ }^{1} \mathrm{H}$ NMR $\left(400 \mathrm{MHz}, 25^{\circ} \mathrm{C}\right): \delta 7.97\left(\mathrm{~d}, 3 \mathrm{H},{ }^{3} J_{\mathrm{HH}}=8.24 \mathrm{~Hz}, 5,10,15-o 1-\mathrm{Ph}\right)$; $7.64\left(\mathrm{~d}, 2 \mathrm{H},{ }^{3} J_{\mathrm{HH}}=7.96 \mathrm{~Hz}, 5,15-o 2-\mathrm{Ph}\right) ; 7.60\left(\mathrm{~d}, 2 \mathrm{H},{ }^{3} J_{\mathrm{HH}}=7.68 \mathrm{~Hz}, 5,15-m 1-\mathrm{Ph}\right) ; 7.55\left(\mathrm{~d}, 1 \mathrm{H},{ }^{3} J_{\mathrm{HH}}=8.92 \mathrm{~Hz}\right.$, $10-o 2-\mathrm{Ph}) ; 7.49\left(\mathrm{~d}, 3 \mathrm{H},{ }^{3} \mathrm{~J}_{\mathrm{HH}}=7.72 \mathrm{~Hz}, 10-m 1 \& 5,15-m 2-\mathrm{Ph}\right) ; 7.43\left(\mathrm{~d}, 1 \mathrm{H},{ }^{3} J_{\mathrm{HH}}=7.72 \mathrm{~Hz}, 10-m 2-\mathrm{Ph}\right) ; 2.72(\mathrm{~s}, 6 \mathrm{H}$, 5,15-p- $\left.\mathrm{CH}_{3}\right) ; 2.69$ (s, 3H, 10-p- $\mathrm{CH}_{3}$ ). MS (ESI): Elemental analysis calcd for $\mathrm{C}_{40} \mathrm{H}_{21} \mathrm{ON}_{4} \mathrm{Cl}_{8} \mathrm{Re}: \mathrm{C} 46.04, \mathrm{H} 2.03$, N 5.37; found: C 46.11, H 1.67, N 5.60. MS (ESI): $\mathrm{M}^{+}=1041.8746$ (expt), 1041.8728 (calcd for $\mathrm{C}_{40} \mathrm{H}_{21} \mathrm{ON}_{4} \mathrm{Cl}_{8} \mathrm{Re}$ ).

General procedure for the synthesis of $\operatorname{Re}\left[\mathrm{Br}_{8} T p X P C\right](O)$ and $\operatorname{Re}\left[\mathrm{Br}_{11} T p X P C\right](O)$. To a chloroform solution of $\operatorname{Re}[\mathrm{T} p \mathrm{XPC}](\mathrm{O})\left(10 \mathrm{~mL} ; \mathrm{X}=\mathrm{F}: 8 \mathrm{mg}, 0.0102 \mathrm{mmol} ; \mathrm{X}=\mathrm{H}: 16 \mathrm{mg}, 0.022 \mathrm{mmol} ; \mathrm{X}=\mathrm{CH}_{3}: 10 \mathrm{mg}\right.$, $0.013 \mathrm{mmol}$, and $\mathrm{X}=\mathrm{OCH}_{3}: 15 \mathrm{mg}, 0.018 \mathrm{mmol}$ ) was added a solution of liquid bromine (a total of 300 equiv) solution in chloroform in three $10-\mathrm{mL}$ portions (each containing 100 equiv $\mathrm{Br}_{2}$ and added over $20 \mathrm{~min}$ ) at 24-h intervals over 3 days. The reaction was then allowed to proceed under stirring at room temperature for a total of 7 days. The resulting mixture was quenched by washing with $20 \%$ aqueous sodium metabisulfite $(25 \mathrm{~mL} \times 3)$. The organic phase was then washed with distilled water $(50 \mathrm{~mL})$, dried over sodium sulfate, and rotary evaporated to dryness. The crude reaction mixture was loaded onto a silica gel column prepared with 4:1 hexane/dichloromethane and eluted with the same solvent system. The greenish-red product was rotary evaporated to dryness and further purified via preparative thin-layer chromatography with the same eluent. Detailed analytical and respective yield of the compounds synthesized are given below. X-ray-quality crystals of $\operatorname{Re}\left[\mathrm{Br}_{8} \mathrm{~T} p \mathrm{FPC}\right](\mathrm{O})$ were obtained by slow diffusion of methanol vapor into a concentrated benzene solution of the complex.

$\operatorname{Re}\left[\mathrm{Br}_{8} \mathrm{TpFPC}\right](\mathrm{O})$. Yield $9 \mathrm{mg}(62.09 \%)$. UV-Vis $\left(\mathrm{CH}_{2} \mathrm{Cl}_{2}\right) \lambda_{\max }\left[\mathrm{nm}, \varepsilon \times 10^{-4}\left(\mathrm{M}^{-1} \mathrm{~cm}^{-1}\right)\right]: 357$ (2.08), 456 (9.77), 574 (1.43), 607 (2.12). ${ }^{1} \mathrm{H}$ NMR (400 MHz, $\left.25^{\circ} \mathrm{C}\right): \delta 8.11(\mathrm{~m}, 1 \mathrm{H}, 10-o 1-\mathrm{Ph}) ; 8.05$ (m, 2H, 5, 15-o1-Ph); $7.78\left(\mathrm{~m}, 2 \mathrm{H},{ }^{3} J_{\mathrm{HH}}=7.68 \mathrm{~Hz}, 5,15-o 2-\mathrm{Ph}\right) ; 7.65(\mathrm{~m}, 1 \mathrm{H}, 10-\mathrm{o} 2-\mathrm{Ph}) ; 7.55-7.36(\mathrm{~m}, 6 \mathrm{H}, 5,10,15-m 1 \& m 2-\mathrm{Ph})$. Elemental analysis calcd for $\mathrm{C}_{37} \mathrm{H}_{12} \mathrm{~F}_{3} \mathrm{ON}_{4} \mathrm{Br}_{8}$ Re: $\mathrm{C} 31.50, \mathrm{H} 0.86, \mathrm{~N} 3.97$; found: $\mathrm{C} 31.78, \mathrm{H} 1.24, \mathrm{~N}$ 3.68. MS (ESI): $\mathrm{M}^{+}=1411.3885$ (expt), 1411.3889 (calcd for $\mathrm{C}_{37} \mathrm{H}_{12} \mathrm{~F}_{3} \mathrm{ON}_{4} \mathrm{Br}_{8} \mathrm{Re}$ ).

$\operatorname{Re}\left[\mathrm{Br}_{8} \mathrm{TPC}\right](\mathrm{O})$. Yield $20 \mathrm{mg}(66.76 \%)$. UV-Vis $\left(\mathrm{CH}_{2} \mathrm{Cl}_{2}\right) \lambda_{\max }\left[\mathrm{nm}, \varepsilon \times 10^{-4}\left(\mathrm{M}^{-1} \mathrm{~cm}^{-1}\right)\right]: 357$ (2.28), 456 (10.45), 575 (1.59), 607 (2.40). ${ }^{1} \mathrm{H}$ NMR (400 MHz, $\left.25^{\circ} \mathrm{C}\right): \delta 8.14$ (d, $\left.1 \mathrm{H},{ }^{3} J_{\mathrm{HH}}=7.48 \mathrm{~Hz}, 10-o 1-\mathrm{Ph}\right) ; 8.08(\mathrm{~d}$, $\left.2 \mathrm{H},{ }^{3} \mathrm{~J}_{\mathrm{HH}}=7.40 \mathrm{~Hz}, 5,15-o 1-\mathrm{Ph}\right) ; 7.86-7.62(\mathrm{~m}, 12 \mathrm{H}, \mathrm{Ph})$. Elemental analysis calcd for $\mathrm{C}_{37} \mathrm{H}_{15} \mathrm{ON}_{4} \mathrm{Br}_{8} \mathrm{Re} \mathrm{C}$ 32.75, H 1.11, N 4.13; found: C 32.49, H 0.95, N 3.88. MS (ESI): $\mathrm{M}^{+}=1357.4166$ (expt), 1357.4174(calcd for $\left.\mathrm{C}_{37} \mathrm{H}_{15} \mathrm{ON}_{4} \mathrm{Br}_{8} \mathrm{Re}\right)$.

$\operatorname{Re}\left[\mathrm{Br}_{11} \mathrm{~T} p \mathrm{CH}_{3} \mathrm{PC}\right](\mathrm{O})$. Yield $12 \mathrm{mg}$ (56.36\%). UV-Vis $\left(\mathrm{CH}_{2} \mathrm{Cl}_{2}\right) \lambda_{\max }\left[\mathrm{nm}, \varepsilon \times 10^{-4}\left(\mathrm{M}^{-1} \mathrm{~cm}^{-1}\right)\right]: 359$ (2.07), 458 (9.70), 574 (1.44), 608 (2.10). ${ }^{1} \mathrm{H}$ NMR $\left(400 \mathrm{MHz}, 25^{\circ} \mathrm{C}\right): \delta 8.35$ (s, $\left.1 \mathrm{H}, \mathrm{Ph}\right) ; 8.28(\mathrm{~s}, 1 \mathrm{H}, \mathrm{Ph}) ; 8.02(\mathrm{~s}, 1 \mathrm{H}$, $\mathrm{Ph}) ; 7.97\left(\mathrm{~d}, 1 \mathrm{H},{ }^{3} J_{\mathrm{HH}}=7.68 \mathrm{~Hz}, \mathrm{Ph}\right) ; 7.92\left(\mathrm{~d}, 2 \mathrm{H},{ }^{3} J_{\mathrm{HH}}=8.44 \mathrm{~Hz}, \mathrm{Ph}\right) ; 7.68\left(\mathrm{~d}, 3 \mathrm{H},{ }^{3} J_{\mathrm{HH}}=8.08 \mathrm{~Hz}, \mathrm{Ph}\right) ; 7.59(\mathrm{~d}$, $\left.1 \mathrm{H},{ }^{3} J_{\mathrm{HH}}=7.84 \mathrm{~Hz}, \mathrm{Ph}\right) ; 7.53(\mathrm{~s}, 1 \mathrm{H}, \mathrm{Ph}) ; 2.78\left(\mathrm{~s}, 6 \mathrm{H}, 5,15-\mathrm{CH}_{3}\right) ; 2.72\left(\mathrm{~s}, 3 \mathrm{H}, 10-p \mathrm{CH}_{3}\right)$. MS (ESI): Elemental analysis calcd for $\mathrm{C}_{40} \mathrm{H}_{18} \mathrm{ON}_{4} \mathrm{Br}_{11}$ Re: $\mathrm{C} 29.37, \mathrm{H} 1.11, \mathrm{~N} 3.43$; found: $\mathrm{C} 29.59, \mathrm{H} 1.41, \mathrm{~N} 3.33 . \mathrm{M}^{+}=1635.1998$ (expt), 1635.1936(calcd for $\mathrm{C}_{40} \mathrm{H}_{18} \mathrm{ON}_{4} \mathrm{Br}_{11} \mathrm{Re}$ ).

$\operatorname{Re}\left[\mathrm{Br}_{11} \mathrm{TpOCH}_{3} \mathrm{PC}\right](\mathrm{O})$. Yield $19 \mathrm{mg}(59.81 \%)$. UV-Vis $\left(\mathrm{CH}_{2} \mathrm{Cl}_{2}\right) \lambda_{\max }\left[\mathrm{nm}, \varepsilon \times 10^{-4}\left(\mathrm{M}^{-1} \mathrm{~cm}^{-1}\right)\right]: 356$ (1.82), 459 (8.59), 574 (1.15), 608 (1.72). ${ }^{1} \mathrm{H}$ NMR (400 MHz, $\left.25^{\circ} \mathrm{C}\right): \delta 8.34$ (s, $\left.1 \mathrm{H}, \mathrm{Ph}\right) ; 8.28$ (s, 1H, Ph); $8.01(\mathrm{~s}, 1 \mathrm{H}$, 
$\mathrm{Ph}) ; 7.71\left(\mathrm{~d}, 2 \mathrm{H},{ }^{3} J_{\mathrm{HH}}=8.72 \mathrm{~Hz}, \mathrm{Ph}\right) ; 7.57\left(\mathrm{~d}, 2 \mathrm{H},{ }^{3} J_{\mathrm{HH}}=8.36 \mathrm{~Hz}, \mathrm{Ph}\right) ; 7.35\left(\mathrm{~d}, 3 \mathrm{H},{ }^{3} J_{\mathrm{HH}}=9.32 \mathrm{~Hz}, \mathrm{Ph}\right) ; 7.19$ $\left(\mathrm{d}, 1 \mathrm{H},{ }^{3} \mathrm{~J}_{\mathrm{HH}}=8.20 \mathrm{~Hz}, \mathrm{Ph}\right) ; 4.23\left(\mathrm{~s}, 6 \mathrm{H}, 5,15-\mathrm{OCH}_{3}\right) ; 4.18\left(\mathrm{~s}, 3 \mathrm{H}, 10-\mathrm{OCCH}_{3}\right)$. Elemental analysis calcd for $\mathrm{C}_{40} \mathrm{H}_{18} \mathrm{O}_{4} \mathrm{~N}_{4} \mathrm{Br}_{11}$ Re: $\mathrm{C} 28.53, \mathrm{H} 1.08, \mathrm{~N} 3.33$; found: C 28.17, H 1.35, N 2.98. MS (ESI): $\mathrm{M}^{+}=1683.1775$ (expt), 1683.1784(calcd for $\mathrm{C}_{40} \mathrm{H}_{18} \mathrm{O}_{4} \mathrm{~N}_{4} \mathrm{Br}_{11} \mathrm{Re}$ ).

X-ray structure determinations. All X-ray diffraction data were collected on beamline 12.2.1 at the Advanced Light Source of Lawrence Berkeley National Laboratory, Berkeley, California. The samples were mounted on MiTeGen kapton loops and placed in a 100(2) K nitrogen cold stream provided by an Oxford Cryostream 700 Plus low temperature apparatus on the goniometer head of a Bruker D8 diffractometer equipped with PHOTONII CPAD detector. Diffraction data were collected using synchrotron radiation monochromated with silicon(111) to a wavelength of 0.7288 (1) Å. In each case, an approximate full-sphere of data was collected using $1^{\circ} \phi$ and $\omega$ scans. Absorption corrections were applied using SADABS ${ }^{73}$. The structure was solved by intrinsic phasing (SHELXT) ${ }^{74}$ and refined by full-matrix least squares on $F^{2}$ (SHELXL-2014) ${ }^{75}$ using the ShelXle $\mathrm{GUI}^{76}$. Appropriate scattering factors were applied using the XDISP ${ }^{77}$ program within the WinGX suite ${ }^{78}$. All non-hydrogen atoms were refined anisotropically. Hydrogen atoms were geometrically calculated and refined as riding atoms.

\section{Accession codes}

The crystal structures described in this paper have been deposited at the Cambridge Crystallographic Data Centre and been assigned the following deposition numbers CCDC 1532043-1532045.

Received: 30 July 2020; Accepted: 27 October 2020

Published online: 12 November 2020

\section{References}

1. Traylor, T. G. \& Tsuchiya, S. Perhalogenated tetraphenylhemins: stable catalysts of high turnover catalytic hydroxylations. Inorg. Chem. 26, 1338-1339 (1987).

2. Dolphin, D., Traylor, T. G. \& Xie, L. Y. Polyhaloporphyrins: unusual ligands for metals and metal-catalyzed oxidations. Acc. Chem. Res. 30, 251-259 (1997).

3. Grinstaff, M. W., Hill, M. G., Labinger, J. A. \& Gray, H. B. Mechanism of catalytic oxygenation of alkanes by halogenated iron porphyrins. Science 264, 1311-1313 (1994).

4. Lyons, J. E., Ellis, P. E. \& Myers, H. K. Halogenated metalloporphyrin complexes as catalysts for selective reactions of acyclic alkanes with molecular oxygen. J. Catal. 155, 59-73 (1995).

5. Groves, J. T., Shalyaev, K. V., Bonchio, M. \& Carofiglio, T. Rapid catalytic oxygenation of hydrocarbons with perhalogenated ruthenium porphyrin complexes. Stud. Surf. Sci. Catal. 110, 865-872 (1997).

6. Costas, M. Selective C-H oxidation catalyzed by metalloporphyrins. Coord. Chem. Rev. 255, 2912-2932 (2011).

7. Mandon, D. et al. $\beta$-Halogenated-pyrrole porphyrins. Molecular structures of 2,3,7,8,12,13,17,18-octabromo-5,10,15,20-tetramesitylporphyrin, nickel(II) 2,3,7,8,12,13,17,18-octabromo-5,10,15,20-tetramesitylporphyrin, and nickel(II) 2,3,7,8,12,13,17,18-octabromo-5,10,15,20-tetrakis(pentafluorophenyl)porphyrin. Inorg. Chem. 31, 2044-2049 (1992).

8. Grinstaff, M. W. et al. Structures, electronic properties, and oxidation-reduction reactivity of halogenated iron porphyrins. Inorg. Chem. 34, 4896-4902 (1995).

9. Birnbaum, E. R. et al. ${ }^{19} \mathrm{~F}$ NMR spectra and structures of halogenated porphyrins. Inorg. Chem. 34, 3625-3632 (1995).

10. Thomassen, I. K., Vazquez-Lima, H., Gagnon, K. J. \& Ghosh, A. Octaiodoporphyrin. Inorg. Chem. 54, 11493-11497 (2015).

11. Ochsenbein, P. et al. Conformational effects on the redox potentials of tetraarylporphyrins halogenated at the $\beta$-pyrrole positions. Angew. Chem. Int. Ed. 33, 348-350 (1994).

12. Bhyrappa, P. \& Krishnan, V. Octabromotetraphenylporphyrin and its metal derivatives: Electronic structure and electrochemical properties. Inorg. Chem. 30, 239-245 (1991).

13. Ghosh, A. et al. Electrochemistry of nickel and copper $\beta$-octahalogeno-meso-tetraarylporphyrins. Evidence for important role played by saddling-induced metal(dx2-y2)-porphyrin("a2u") orbital interactions. J. Phys. Chem. B 105, 8120-8124 (2001).

14. Kadish, K. M. \& Van Caemelbecke, E. Electrochemistry of metalloporphyrins in nonaqueous media. In Encyclopedia of Electrochemistry Bioelectrochemistry Vol. 9 (ed. Wilson, G. S.) 175-228 (Wiley-VCH, Weinheim, 2002).

15. Shao, J., Steene, E., Hoffman, B. M. \& Ghosh, A. EPR, ENDOR, and DFT studies on ( $\beta$-Octahalo-meso-tetraarylporphyrin)copper complexes: characterization of the metal $\left(\mathrm{d}_{\mathrm{x} 2-\mathrm{y} 2}\right)$-porphyrin $\left(\mathrm{a}_{2 \mathrm{u}}\right)$ orbital interaction. Eur. J. Inorg. Chem. 2005, 1609-1615 (2005).

16. Gross, Z., Galili, N. \& Saltsman, I. The first direct synthesis of corroles from pyrrole. Angew. Chem. Int. Ed. 38, 1427-1429 (1999).

17. Paolesse, R. et al. 5,10,15-Triphenylcorrole: a product from a modified Rothemund reaction. Chem. Commun. 14, 1307-1308 (1999).

18. Ghosh, A. A perspective of pyrrole-aldehyde condensations as versatile self-assembly processes. Angew. Chem. Int. Ed. 43, 19181931 (2004).

19. Orłowski, R., Gryko, D. \& Gryko, D. T. Synthesis of corroles and their heteroanalogs. Chem. Rev. 117, 3102-3137 (2017).

20. Ghosh, A. Electronic structure of corrole derivatives: insights from molecular structures, spectroscopy, electrochemistry, and quantum chemical calculations. Chem. Rev. 117, 3798-3881 (2017).

21. Nardis, S., Mandoj, F., Stefanelli, M. \& Paolesse, R. Metal complexes of corrole. Coord. Chem. Rev. 388, 360-405 (2019).

22. Golubkov, G. et al. High-valent manganese corroles and the first perhalogenated metallocorrole catalyst. Angew. Chem. Int. Ed. 40, 2132-2134 (2001).

23. Palmer, J. H., Durrell, A. C., Gross, Z., Winkler, J. R. \& Gray, H. B. Iridium corroles. J. Am. Chem. Soc. 130, 7786-7787 (2008).

24. Wasbotten, I. H., Wondimagegn, T. \& Ghosh, A. Electronic absorption, resonance raman, and electrochemical studies of planar and saddled copper(III) meso-triarylcorroles. Highly substituent-sensitive soret bands as a distinctive feature of high-valent transition metal corroles. J. Am. Chem. Soc. 124, 8104-8116 (2002).

25. Alemayehu, A. B., Hansen, L. K. \& Ghosh, A. Nonplanar, noninnocent, and chiral: a strongly saddled metallocorrole. Inorg. Chem. 49, 7608-7610 (2010).

26. Capar, C., Thomas, K. E. \& Ghosh, A. Reductive demetalation of copper corroles: first simple route to free-base $\beta$-octabromocorroles. J. Porphyrins Phthalocyanines 12, 964-967 (2008).

27. Capar, C., Hansen, L.-K., Conradie, J. \& Ghosh, A. $\beta$-octabromo-meso-tris(pentafluorophenyl)corrole: reductive demetalationbased synthesis of a heretofore inaccessible, perhalogenated free-base corrole. J. Porphyrins Phthalocyanines 14, 509-512 (2010).

28. Capar, J. et al. Improved syntheses of $\beta$-octabromo-meso-triarylcorrole derivatives. J. Inorg. Biochem. 153, 162-166 (2015).

29. Rabinovitch, E., Goldberg, I. \& Gross, Z. Gold(I) and gold(III) corroles. Chem. Eur. J. 17, 12294-12301 (2011). 
30. Thomas, K. E., Gagnon, K. J., McCormick, L. J. \& Ghosh, A. Molecular structure of gold 2,3,7,8,12,13,17,18-octabromo-5,10,15tris(4"-pentafluorosulfanylphenyl)corrole: Potential insights into the insolubility of gold octabromocorroles. J. Porphyrins Phthalocyanines 22, 596-601 (2018).

31. Norehim, H.-K. et al. Ligand noninnocence in FeNO corroles: insights from $\beta$-octabromocorrole complexes. Dalton Trans. 45, 681-689 (2018).

32. Alemayehu, A. B., Gonzalez, E., Hansen, L. K. \& Ghosh, A. Copper corroles are inherently saddled. Inorg. Chem. 48, 7794-7799 (2009).

33. Thomas, K. E., Conradie, J., Hansen, L. K. \& Ghosh, A. A metallocorrole with orthogonal pyrrole rings. Eur. J. Inorg. Chem. 2011, 1865-1870 (2011).

34. Berg, S., Thomas, K. E., Beavers, C. M. \& Ghosh, A. Undecaphenylcorroles. Inorg. Chem. 51, 9911-9916 (2012).

35. Thomas, K. E. et al. Halterman corroles and their use as a probe of the conformational dynamics of the inherently chiral copper corrole chromophore. Inorg. Chem. 57, 4270-4276 (2018).

36. Thomassen, I. K., McCormick, L. J. \& Ghosh, A. Synthesis and molecular structure of a copper octaiodocorrole. ACS Omega 3, 5106-5110 (2018).

37. Thomas, K. E., Settineri, N. S., Teat, S. J., Steene, E. \& Ghosh, A. Molecular structure of copper and $\mu$-oxodiiron octafluorocorrole derivatives: insights into ligand noninnocence. ACS Omega 5, 10176-10182 (2020).

38. Thomas, K. E. et al. Ligand noninnocence in coinage metal corroles: a silver knife-edge. Chem. Eur. J. 21, 16839-16847 (2015).

39. Alemayehu, A. B. \& Ghosh, A. Gold corroles. J. Porphyrins Phthalocyanines 15, 106-110 (2011).

40. Thomas, K. E., Alemayehu, A. B., Conradie, J., Beavers, C. \& Ghosh, A. Synthesis and molecular structure of gold triarylcorroles. Inorg. Chem. 50, 12844-12851 (2011).

41. Thomas, K. E., Beavers, C. M. \& Ghosh, A. Molecular structure of a gold $\beta$-octakis(trifluoromethyl)-meso-triarylcorrole: an $85^{\circ}$ difference in saddling dihedral relative to copper. Mol. Phys. 110, 2439-2444 (2012).

42. Capar, J. et al. Demetalation of Copper undecaarylcorroles: molecular structures of a free-base undecaarylisocorrole and a gold undecaarylcorrole. J. Inorg. Biochem. 162, 146-153 (2016).

43. Brückner, C., Briñas, R. P. \& Bauer, J. A. K. X-ray structure and variable temperature nmr spectra of [meso-Triarylcorrolato] copper(III). Inorg. Chem. 42, 4495-4497 (2003).

44. Steene, E., Dey, A. \& Ghosh, A. $\beta$-octafluorocorroles. J. Am. Chem. Soc. 125, 16300-16309 (2003).

45. Bröring, M., Brégier, F., Tejero, E. C., Hell, C. \& Holthausen, M. C. Revisiting the electronic ground state of copper corroles. Angew. Chem. Int. Ed. 46, 445-448 (2007).

46. Lim, H. et al. X-ray absorption spectroscopy as a probe of ligand noninnocence in metallocorroles: the case of copper corroles. Inorg. Chem. 58, 6722-6730 (2019).

47. Buckley, H. L. \& Arnold, J. Recent developments in out-of-plane metallocorrole chemistry across the periodic table. Dalton Trans. 44, 30-36 (2015).

48. Ziegler, J. A., Buckley, H. L. \& Arnold, J. Synthesis and reactivity of tantalum corrole complexes. Dalton Trans. 46, 780-785 (2017).

49. Alemayehu, A. B., Vazquez-Lima, H., Gagnon, K. J. \& Ghosh, A. Tungsten biscorroles: new chiral sandwich compounds. Chem. Eur. J. 22, 6914-6920 (2016).

50. Einrem, R. F., Gagnon, K. J., Alemayehu, A. B. \& Ghosh, A. Metal-ligand misfits: facile access to rhenium-oxo corroles by oxidative metalation. Chem. Eur. J. 22, 517-520 (2016).

51. Alemayehu, A. B., Teat, S. J., Borisov, S. M. \& Ghosh, A. Rhenium-imido corroles. Inorg. Chem. 59, 6382-6389 (2020).

52. Alemayehu, A. B., Gagnon, K. J., Terner, J. \& Ghosh, A. Oxidative metalation as a route to size-mismatched macrocyclic complexes: osmium corroles. Angew. Chem. Int. Ed. 53, 14411-14414 (2014).

53. Alemayehu, A. B. et al. Platinum corroles. Chem. Commun. 50, 11093-11096 (2014).

54. Alemayehu, A. B., McCormick, L. J., Gagnon, K. J., Borisov, S. M. \& Ghosh, A. Stable platinum(IV) corroles: synthesis, molecular structure, and room-temperature near-IR phosphorescence. ACS Omega 3, 9360-9368 (2018).

55. Palmer, J. H., Durrell, A. C., Gross, Z., Winkler, J. R. \& Gray, H. B. Near-IR phosphorescence of iridium(III) corroles at ambient temperature. J. Am. Chem. Soc. 132, 9230-9231 (2010).

56. Sinha, W., Ravotto, L., Ceroni, P. \& Kar, S. NIR-emissive iridium(III) corrole complexes as efficient singlet oxygen sensitizers. Dalton Trans. 44, 17767-17773 (2015).

57. Borisov, S. M., Alemayehu, A. \& Ghosh, A. Osmium-nitrido corroles as NIR indicators for oxygen sensors and triplet sensitizers for organic upconversion and singlet oxygen generation. J. Mater. Chem. C 4, 5822-5828 (2016).

58. Lemon, C. M., Powers, D. C., Brothers, P. J. \& Nocera, D. G. Gold corroles as near-IR phosphors for oxygen sensing. Inorg. Chem. 56, 10991-10997 (2017).

59. Alemayehu, A. B. et al. Gold tris(carboxyphenyl)corroles as multifunctional materials: room temperature near-ir phosphorescence and applications to photodynamic therapy and dye-sensitized solar cells. ACS Appl. Mater. Interfaces 8, 18935-18942 (2016).

60. Borisov, S. M., Einrem, R. F., Alemayehu, A. B. \& Ghosh, A. Ambient-temperature near-IR phosphorescence and potential applications of rhenium-oxo corroles. Photochem. Photobiol. Sci. 18, 1166-1170 (2019).

61. Einrem, R. F., Alemayehu, A. B., Borisov, S. M., Ghosh, A. \& Gederaas, O. A. Amphiphilic rhenium-oxo corroles as a new class of sensitizers for photodynamic therapy. ACS Omega 5, 10596-10601 (2020).

62. Thomassen, I. K., McCormick-McPherson, L. J., Borisov, S. M. \& Ghosh, A. Iridium corroles exhibit weak near-infrared phosphorescence but efficiently sensitize singlet oxygen formation. Sci. Rep. 10, 7551 (2020).

63. Teo, R. D., Hwang, J. Y., Termini, J., Gross, Z. \& Gray, H. B. Fighting cancer with corroles. Chem. Rev. 117, 2711-2729 (2017).

64. Reinholdt, A., Alemayehu, A. B., Gagnon, K. J., Bendix, J. \& Ghosh, A. Electrophilic activation of osmium-nitrido corroles. The OsN triple bond as a $\pi$-acceptor metalla-ligand in a heterobimetallic $\mathrm{Os}^{\mathrm{VI}} \mathrm{N}-\mathrm{Pt}^{\mathrm{II}}$ complex. Inorg. Chem. 59, 5276-5280 (2020).

65. Soll, M. et al. One-pot conversion of fluorophores to phosphorophores. Org. Lett. 18, 5840-5843 (2016).

66. Sudhakar, K. et al. Effect of selective $\mathrm{CF}_{3}$ substitution on the physical and chemical properties of gold corroles. Angew. Chem. Int. Ed. 56, 9837-9841 (2017).

67. Johansen, I. et al. Substituent effects on metallocorrole spectra: insights from chromium-oxo and molybdenum-oxo triarylcorroles. J. Porphyrins Phthalocyanines 15, 1335-1344 (2011).

68. Schweyen, P. et al. Viking helmet corroles: activating inert oxidometal corroles. Chem. Eur. J. 23, 13897-13900 (2017).

69. Mahammed, A., Botoshanskya, M. \& Gross, Z. Chlorinated corroles. Dalton Trans. 41, 10938-10940 (2012).

70. Thomas, K. E., Alemayehu, A. B., Conradie, J., Beavers, C. M. \& Ghosh, A. The structural chemistry of metallocorroles: combined X-ray crystallography and quantum chemistry studies afford unique insights. Acc. Chem. Res. 45, 1203-1214 (2012).

71. Albrett, A. M. et al. Corrole as a binucleating ligand: preparation, molecular structure and density functional theory study of diboron corroles. J. Am. Chem. Soc. 130, 2888-2889 (2008).

72. Albrett, A. M. et al. Mono- and diboron corroles: factors controlling stoichiometry and hydrolytic reactivity. Inorg. Chem. 53, 5486-5493 (2014).

73. Krause, L., Herbst-Irmer, R., Sheldrick, G. M. \& Stalke, D. Comparison of silver and molybdenum microfocus X-ray sources for single-crystal structure determination. J. Appl. Cryst. 48, 3-10 (2015).

74. Sheldrick, G. M. SHELXT-integrated space-group and crystal-structure determination. Acta Cryst. A71, 3-8 (2015).

75. Sheldrick, G. M. Crystal structure refinement with SHELXL. Acta Cryst. C71, 3-8 (2015). 
76. Hübschle, C. B., Sheldrick, G. M. \& Dittrich, B. ShelXle: a Qt graphical user interface for SHELXL. J. Appl. Cryst. 44, 1281-1284 (2011).

77. Kissel, L. \& Pratt, R. H. Corrections to tabulated anomalous-scattering factors. Acta Cryst. A46, 170-175 (1990).

78. Farrugia, L. J. WinGX and ORTEP for windows: an update. J. Appl. Cryst. 45, 849-854 (2012).

\section{Acknowledgements}

We acknowledge Grant No. 262229 (to AG) from the Research Council of Norway. This research used resources of the Advanced Light Source, a U.S. DOE Office of Science User Facility under Contract No. DE-AC02-05CH11231.

\section{Author contributions}

A.B.A. and R.F.E. carried out the synthetic work; L.J.M.M. and N.S.S. carried out the X-ray structure determinations. A.G. planned and supervised the project. The manuscript was largely composed by A.B.A. and A.G.

\section{Competing interests}

The authors declare no competing interests.

\section{Additional information}

Supplementary information is available for this paper at https://doi.org/10.1038/s41598-020-76308-7.

Correspondence and requests for materials should be addressed to A.G.

Reprints and permissions information is available at www.nature.com/reprints.

Publisher's note Springer Nature remains neutral with regard to jurisdictional claims in published maps and institutional affiliations.

(c) (i) Open Access This article is licensed under a Creative Commons Attribution 4.0 International License, which permits use, sharing, adaptation, distribution and reproduction in any medium or format, as long as you give appropriate credit to the original author(s) and the source, provide a link to the Creative Commons licence, and indicate if changes were made. The images or other third party material in this article are included in the article's Creative Commons licence, unless indicated otherwise in a credit line to the material. If material is not included in the article's Creative Commons licence and your intended use is not permitted by statutory regulation or exceeds the permitted use, you will need to obtain permission directly from the copyright holder. To view a copy of this licence, visit http://creativecommons.org/licenses/by/4.0/.

(C) The Author(s) 2020 